\title{
Temperature Field Study and Numerical Simulation of the Power Device of the MOSFET
}

\author{
Xu Jianning ${ }^{\mathrm{a}}$, Feng Lei ${ }^{\mathrm{b}}$, Zhu Duanyin ${ }^{\mathrm{c}}$ \\ Xi'an Shiyou University, Xi'an, Shaanxi province, China \\ a jnxu723@163.com, ${ }^{b}$ flyjw208@126.com, ${ }^{c}$ zhudy@xsyu.edu.cn
}

Keywords: MOSFET, Motor driver, Power loss, Temperature field, Numerical simulation

Abstract: This paper is based on the principle of electric drive automatic control system and the working principle of power electronics technology _MOSFET (Metal-Oxide-Semiconductor Field Effect Transistor), introduced the application of the power device MOSFET in the motor driver and the power loss calculation method of the power device of the MOSFET. Use the finite element software ANSYS/Fluent module for numerical simulation, in addition, the heat conduction process of the energy loss produced by the MOSFET as the inverter power device of the motor driver is discussed. The influence of the height and width of the channel section on the temperature rise of the MOSFET is obtained by changing the size of the cooling channel.

\section{Introduction}

The reliability and performance of power modules have a major impact on motor inverter performance in electric vehicle applications [1]. Infineon's main business: Insulated Gate Bipola Transistor (abbreviated as IGBT), HybridPACKTM1 provides hardware support for the development of complete inverters for electric vehicles with IGBTs in electric vehicle applications. However, the DC busbar is packaged in the module and the heat is difficult to handle and cannot be changed. At the same time, new energy sources in the Chinese market are booming, out of stock and prices are high.

Inverter can realize inverter through IGBT and MOSFET, and the multi-tube parallel connection of IGBT and MOSFET discrete devices can realize the Infineon IGBT module - HybridPACKTM1 inverter function. The motor inverter is the core component of pure electric vehicle development, and the heat treatment of the core power device of the motor inverter directly affects the number of Mosfet used and the safety of the whole machine. In order to improve the efficiency and safety of Mosfet, we conduct thermal analysis of the power Mosfet and optimize the design of the heat dissipation scheme. Using finite element numerical simulation, it can be judged whether the design can meet the thermal reliability of the product before the prototype is produced, and the heat distribution of the power MOSFET can be more visualized, and the heat distribution and heat transfer can be intuitively described. Compared with traditional experiments, it is more low-carbon and saves money. 


\section{Calculation method of MOSFET heat loss based on vehicle inverter}

\subsection{MOSFET Loss Model}

When Phase A of the inverter output is positive, current will follow one of two paths depending on whether S1 is ON or OFF [1]. When S1 is ON, current will flow from +VDC/2 through S1 and return to $-\mathrm{VDC} / 2$ via S2 and S6, seen in Fig.1. Since current is flowing through the MOSFET, resistance in the silicon will cause MOSFET conduction loss[2]. When S1 is turned OFF, the windings of the stator will continue to draw current. Since current can no longer flow through S1, it will freewheel through the protection diode of S4, seen in Fig.2 During this period, resistance in the p-n junction of the diode will cause an additional Diode conduction loss[3]. The MOSFET of S4 will not be switching, thus not experiencing conduction loss, and the diode of S1 will not be forward biased, thus not experiencing conduction loss, while Phase A is positive. When Phase A of the inverter output current becomes negative, the roles of S1 and S4 are reversed - S1 will experience Diode conduction loss and S4 will experience MOSFET conduction loss. Therefore one sixth of the combined conduction loss for one fundamental period can be calculated by adding the MOSFET conduction loss of S1 when Phase A is positive to the Diode conduction loss of S1 when Phase A in negative [4].

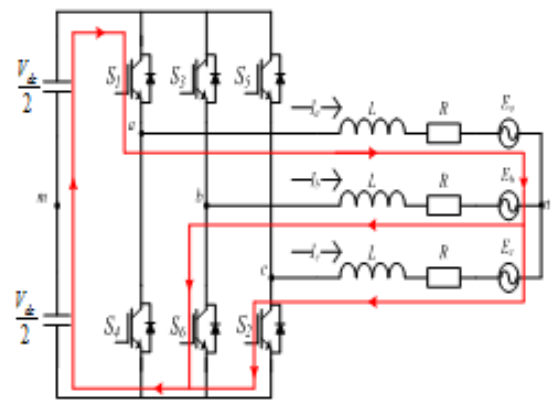

Figure 1 Current path when $S_{1}$ is on

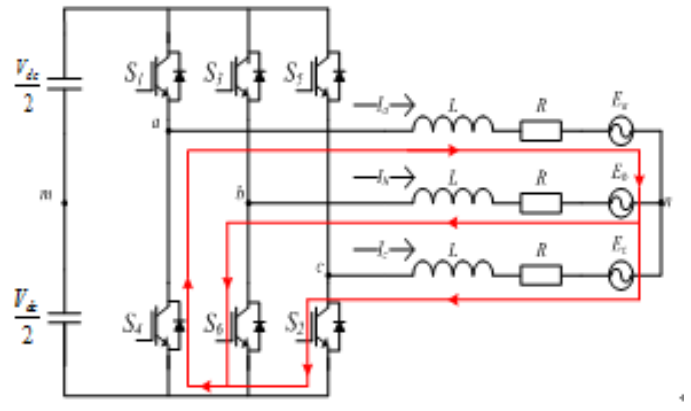

Figure 2 Current path when $\mathrm{S}_{1}$ is off

Similarly, when Phase A of the inverter output current is positive, the IGBT of $S_{1}$ will be switching between ON and OFF while the protection diode of $\mathrm{S}_{4}$ will be switching between forward and reverse bias[5]. When IGBT switches ON, current begins to flow as the voltage drop VCE shrinks. Since the channel in the IGBT does not form instantaneously, the resistance drops to its ON value as a function of time. Likewise, when the IGBT is turned off, the channel does not disperse immediately and electron recombination causes the resistance to increase as a slower function of time than turn-on. Energy loss during turn-on and turn-off is the switching loss[6].

\subsection{MOSFET Conduction Loss Model}

Static conduction loss uses a linear approximation of the $I_{C}\left(V_{d s}\right)$ plot in the MOSFET data sheet. The inverse of the slope and x-intercept of the linearization are $R_{d s}$ and $V_{d s}$ respectively. The static conduction loss equation is as follows[7]:

$$
P_{\text {loss }}=\left[\frac{1}{T} \int_{0}^{t 1} i_{C}(t) d t * V_{C E 0}\right]+\left[R_{\text {on }} * \frac{1}{T} \int_{0}^{t 1} i_{C}(t)^{2} d t\right]=I_{\text {avg }} V_{C E 0}+I_{R M S}{ }^{2} R_{o n}
$$

Where: $\mathrm{T}=$ Fundamental Period; $\mathrm{t} 1=$ portion of $\mathrm{T}$ that $\mathrm{I}_{\mathrm{a}-\text { average }}$ is positive

Dynamic conduction loss uses two-dimensional linear interpolation between, and extrapolation from, data points taken at MOSFET data sheet. This is accomplished through the use of the 2D lookup table function in MatLab. The dynamic conduction loss equation is as follows: 


$$
P_{\text {loss }}=i_{C}(t) * v_{C E}\left(i_{C}(t), T_{j}\right)
$$

Where: $\mathrm{T}_{\mathrm{j}}=$ Junction Temperature

\subsection{Diode Conduction Loss Model}

Static conduction loss uses a linear approximation of the $\mathrm{I}_{\mathrm{F}}\left(\mathrm{V}_{\mathrm{F}}\right)$ plot in the MOSFET data sheet. The inverse of the slope and $\mathrm{x}$-intercept of the linearization are $\mathrm{R}_{\mathrm{on} \_\mathrm{d}}$ and $\mathrm{V}_{\mathrm{F} 0}$ respectively. The static conduction loss equation is as follows[8]:

$$
\mathrm{P}_{\text {loss }}=\left[\frac{1}{\mathrm{~T}} \int_{\mathrm{t} 1}^{\mathrm{T}} \mathrm{i}_{\mathrm{F}}(\mathrm{t}) \mathrm{dt} * \mathrm{~V}_{\mathrm{F} 0}\right]+\left[\mathrm{R}_{\mathrm{on} \_\mathrm{d}} * \frac{1}{\mathrm{~T}} \int_{\mathrm{t} 1}^{\mathrm{T}} \mathrm{i}_{\mathrm{F}}(\mathrm{t})^{2} \mathrm{dt}\right]=\mathrm{I}_{\mathrm{avg}} \mathrm{V}_{\mathrm{F} 0}+\mathrm{I}_{\mathrm{RMS}}{ }^{2} \mathrm{R}_{\mathrm{on} \_\mathrm{d}}
$$

Where: $\mathrm{T}=$ Fundamental Period; $\mathrm{t} 1=$ portion of $\mathrm{T}$ that $\mathrm{I}_{\mathrm{a} \_ \text {average }}$ is positive; for $\mathrm{T}-\mathrm{t}$, $\mathrm{I}_{\mathrm{a} \_ \text {average }}$ is negative

Dynamic conduction loss uses two-dimensional linear interpolation between, and extrapolation from, data points taken at MOSFET data sheet. This is accomplished through the use of the 2D lookup table function in MatLab. The dynamic conduction loss equation is as follows:

$$
\mathrm{P}_{\text {loss }}=\mathrm{i}_{\mathrm{F}}(\mathrm{t}) * \mathrm{v}_{\mathrm{CE}}\left(\mathrm{i}_{\mathrm{F}}(\mathrm{t}), \mathrm{T}_{\mathrm{j}}\right)
$$

Where: $\mathrm{T}_{\mathrm{j}}=$ Junction Temperature

\subsection{MOSFET Switching Loss and Diode Reverse Recovery Model}

To calculate the switching power loss, we first calculate the one-pulse turn on and turn off switching energy loss Esw_on_ins and Esw_off_ins in each switching period. Esw_on_ins and Esw_off_ins are calculated based on the MOSFET datasheet[9].

$$
\begin{array}{r}
\mathrm{E}_{\mathrm{sw} \_o n \_ \text {ins }}=\mathrm{E}_{\mathrm{on}} *\left(\mathrm{I}_{\mathrm{a} \_ \text {on }} * \mathrm{~V}_{\mathrm{on}}\right) /(550 * 300) \\
\mathrm{E}_{\mathrm{s} \mathrm{s}_{\text {off }} \text { ins }}=\mathrm{E}_{\mathrm{off}} *\left(\mathrm{I}_{\mathrm{a} \_ \text {off }} * \mathrm{~V}_{\mathrm{off}}\right) /(550 * 300)
\end{array}
$$

In each switching period, that one pulse turn-on and turn-off losses are calculated. Then add them together to get the total turn on switching energy losses Esw_on and turn off switching energy losses Esw_off in one positive period of phase A current. Finally, using the equation below, the switching power loss is calculated based on the total switching energy loss.

$$
P_{s w}=\left(E_{s w_{-} o n}+E_{\text {Sw_off }}\right) / T_{1}
$$

Where $\mathrm{T}_{1}$ is the fundamental period of the phase current and is calculated

\section{Numerical Simulation of MOSFET Temperature Field}

\subsection{Structure model diagram of MOSFET power board}

According to the real flow characteristics of the surface of the object, the structural shape of the engineering object is simplified, and the related physical model is established [10].

The MOSFET power board structure model is shown in Fig.3, and the water channel cross-sectional area is $12 \times 3 \mathrm{~mm}$. PCB and MOSFET tubes are simplified to solid and equivalent to equivalent material properties. 


\subsection{Solver setup and solution}

The ANSYS Fluent module is used for calculation, and the Realizable k-e turbulence equation is used to process the wall surface with Scalable Wall Function. Finally, the Coupled algorithm is used to solve the problem. The inlet flow rate is $6 \mathrm{Lpm}$, the flow rate per well is $2 \mathrm{Lpm}$, and the inlet temperature is $65^{\circ} \mathrm{C}$. Material properties are shown in Table 1.

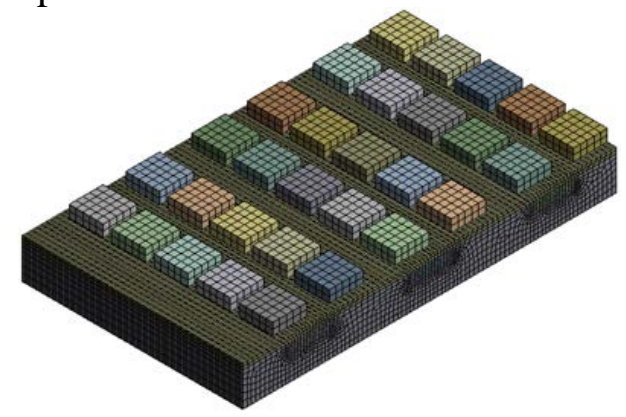

Figure. 3 Structure grid division diagram of MOSFET power board

Table 1 Material Properties

\begin{tabular}{|c|c|c|c|c|c|}
\hline Name & Material name & $\begin{array}{l}\text { Density } \\
\mathrm{kg} / \mathrm{m}^{3}\end{array}$ & $\begin{array}{l}\text { Specific heat } \\
\text { capacity } \\
\text { J/(kg.K) }\end{array}$ & $\begin{array}{c}\text { Thermal } \\
\text { conductivity } \\
\text { W/(m·K) }\end{array}$ & $\begin{array}{l}\text { Viscosity } \\
\mathrm{kg} /(\mathrm{m} \cdot \mathrm{s})\end{array}$ \\
\hline solder & & 7400 & 230 & 28 & \\
\hline MOSFET & $\begin{array}{c}\text { Equivalent } \\
\text { material }\end{array}$ & 2400 & 1000 & 100 & \\
\hline Box & Aluminum 6061 & 2719 & 971 & 167 & \\
\hline Coolant & $\begin{array}{c}50 \% \text { ethylene } \\
\text { glycol aqueous } \\
\text { solution }\end{array}$ & 1046.94 & 3464 & 0.407 & 0.00123 \\
\hline Thermal Grease & & 2800 & 1000 & 1 & \\
\hline PCB & $\begin{array}{c}\text { Equivalent } \\
\text { material }\end{array}$ & 3103 & 861 & $\begin{array}{c}\text { In-plane : } 174.139 \\
\text { Normal direction: } \\
40.7839\end{array}$ & \\
\hline
\end{tabular}

The PCB equivalent properties are calculated using Icepak software. The copper layer has a copper coverage rate of $90 \%$.

Each MOSFET has a loss of 30W, and the thermal resistance RthJC of the MOSFET tube is $0.28^{\circ} \mathrm{C} / \mathrm{W}$. Considering the thermal grease between the PCB and the case, the equivalent thickness is $150 \mathrm{um}$ and the thickness of the solder layer is $0.1 \mathrm{~mm}$.

\subsection{Calculation results and post processing}

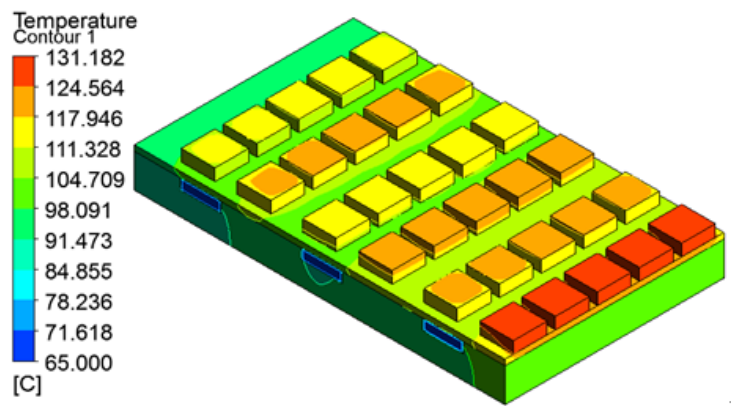

Figure. 4 Steady-state temperature distribution 
The steady-state temperature distribution is shown in Fig.4. When the water channel section is 12 $\times 3 \mathrm{~mm}$, the maximum temperature of the MOSFET tube is $131.182{ }^{\circ} \mathrm{C}$.

The calculation results of different water channel sections are shown in Table 2. The maximum temperature of the MOSFET tube is highly sensitive to the cross section of the water channel, and is not sensitive to the width of the water channel. The smaller the cross-sectional area of the water channel, the greater the pressure loss.

Table 2 Calculation results for different sections

\begin{tabular}{|c|c|c|}
\hline Section(mm) & Pressure loss(Pa) & Maximum temperature of MOSFET $\left({ }^{\circ} \mathbf{C}\right)$ \\
\hline $12 \times 7$ & 26.72 & 165.45 \\
\hline $12 \times 5$ & 68.37 & 147.97 \\
\hline $12 \times 3$ & 268.3 & 131.18 \\
\hline $12 \times 2$ & 783.7 & 122.36 \\
\hline $10 \times 3$ & 382.3 & 131.57 \\
\hline $8 \times 3$ & 591.3 & 131.76 \\
\hline $5 \times 3$ & 1057 & 131.53 \\
\hline
\end{tabular}

\section{Conclusion}

1) The numerical simulation is carried out by the finite element software ANSYS/Fluent module to obtain the internal crystallization temperature of the MOSFET, so as to control the over-temperature failure of the MOSFET from the source.

2) The numerical simulation is carried out by the finite element software ANSYS/Fluent module to obtain the influence of the size of the water channel on the cooling effect of the MOSFET. The MOSFET tube temperature is sensitive to the height of the water channel section, and the width of the water channel section has little effect on the temperature of the MOSFET tube. The smaller the cross-sectional area of the water channel, the greater the pressure loss.

3) The MOSFET multi-tube parallel scheme can replace the MOSFET module scheme by simulation, which provides a more competitive cost advantage for the realization of the new energy vehicle motor inverter.

\section{References}

[1] Stephen D.Umans. Electric Machinery. Seventh Edition.Henry Dream press,2008.

[2] YAN Dawei, CHEN Shiyuan, Performance Compare of Driving Motors on EV, 2nd, Automobile Electric Appliance. 2004, pp. 4-6.

[3] R. M. Warner. MOSFET Theory and Design. USA: Oxford University Press, 1999.

[4] Galup-Montoro, Carlos. Mosfet Modeling for Circuit Analysis and Design. Singapore: WORLD SCIENTIFIC PUBLISHING, 2007.

[5] Kong Xiaoguang, Wang Fengxiang , Xing Junqiang, Losses Calculation and Temperature Field Analysis of High Speed Permanent Magnet Machines, No.9, Vo1.27, Transactions of China Electrotechnical Society. 2012, pp. 166-172.

[6] John H Lienhard IV. A Heat Transfer Textbook. Fourth Edition. Dover Publications,2011.

[7] Xing Junqiang, Wang Fengxiang, Zhang Dianhai, Research on Rotor Air Friction Loss of High-speed Permanent Magnet Machines, No.27, Vol.30, Proceedings of the CSEE. 2010, pp. 14-19.

[8] Xu Yongxiang, Hu Jianhui, Hu Renzhi, Zou Jibin, An Experimental Verification Method of Calculation for Rotor EddyCurrent Losses in PMSMs, No.7, Vo1.22, Transactions of China Electrotechnical Society. 2007, pp.150-154.

[9] Ding Shuye, Liu Shuqi, Bi Liuxin, Loss Analysis for Permanent Magnet Synchronous Motor under Vector Control, No.12, Vo1.47, Journal of Xi'an Jiaotong University. 2013, pp. 95-101.

[10] Yuhua Cheng, ChenmingHu. Mosfet Modeling and Bsim3 User's Guide. Dordrecht: Kluwer Academic Publishers, 1988. 